\title{
Extracts containing CLPs of Bacillus amyloliquefaciens JN68 isolated from chicken intestines exert antimicrobial effects, particularly on methicillin-resistant Staphylococcus aureus and Listeria monocytogenes
}

\author{
JEN-NI CHEN ${ }^{1,2^{*}}$, CHYOU-WEI WEI $^{1,3^{*}}$, HSIAO-CHUN LIU ${ }^{1,4}$, SHU-YING CHEN ${ }^{3,5}$, \\ CHINSHUH CHEN ${ }^{2}$, YU-MIN JUANG ${ }^{6}$, CHIEN-CHEN LAI $^{6,7}$ and GIOU-TENG YIANG ${ }^{8,9}$ \\ ${ }^{1}$ Department of Nutrition, Master Program of Biomedical Nutrition, Hungkuang University, Taichung 433; \\ ${ }^{2}$ Department of Food Science and Biotechnology, National Chung Hsing University, Taichung 402; \\ ${ }^{3}$ Department of Nursing, Hungkuang University, Taichung 433; ${ }^{4}$ Department of Nursing, Taipei Tzu Chi Hospital, \\ Buddhist Tzu Chi Medical Foundation, New Taipei 231; ${ }^{5}$ Department of Biotechnology, Hungkuang University, \\ Taichung 433; ${ }^{6}$ Institute of Molecular Biology, National Chung Hsing University, Taichung 402; \\ ${ }^{7}$ Graduate Institute of Chinese Medical Science, China Medical University, Taichung 404; \\ ${ }^{8}$ Department of Emergency Medicine, School of Medicine, Tzu Chi University, Hualien 970; \\ ${ }^{9}$ Department of Emergency Medicine, Taipei Tzu Chi Hospital, Buddhist Tzu Chi Medical Foundation, \\ New Taipei 231, Taiwan, R.O.C.
}

Received September 27, 2015; Accepted October 11, 2016

DOI: $10.3892 / \mathrm{mmr} .2016 .5900$

\begin{abstract}
Bacillus amyloliquefaciens JN68, which has been discussed with regards to its antimicrobial activities, was successfully isolated from healthy chicken intestines in the present study. Using the spot-on-the-lawn antagonism method, the preliminary study indicated that a suspension culture of the B. amyloliquefaciens JN68 strain can inhibit the growth of Aspergillus niger and Penicillium pinophilum. Furthermore, the cyclic lipopeptides (CLPs) produced by the B. amyloliquefaciens JN68 strain were further purified through acid precipitation and Bond Elut ${ }^{\circledR} \mathrm{C} 18$ chromatography, and their structures were identified using the liquid chromatography-electrospray ionization-mass spectrometry (MS)/MS method. Purified CLPs exerted broad spectrum antimicrobial activities on various pathogenic and foodborne bacteria and fungi, as determined using the agar well diffusion method. Listeria monocytogenes can induce listeriosis, which is associated with a high mortality rate. Methicillin-resistant
\end{abstract}

Correspondence to: Dr Giou-Teng Yiang, Department of Emergency Medicine, School of Medicine, Tzu Chi University, 701, Section 3, Chung Yan Road, Hualien 970, Taiwan, R.O.C.

E-mail: gioutengyiang@gmail.com

*Contributed equally

Key words: Bacillus amyloliquefaciens JN68, antimicrobial, cyclic lipopeptides, methicillin-resistant Staphylococcus aureus
Staphylococcus aureus (MRSA) is a major pathogenic bacteria that causes nosocomial infections. Therefore, L. monocytogenes and MRSA are currently of great concern. The present study aimed to determine whether B. amyloliquefaciens JN68 extracts could inhibit L. monocytogenes and MRSA. The results indicated that extracts of $B$. amyloliquefaciens JN68 have CLP components, and can successfully inhibit the growth of L. monocytogenes and MRSA.

\section{Introduction}

B. amyloliquefaciens is widely present in soil, plants and some animals (1-6); therefore, up to now, several strains of B. amyloliquefaciens have been isolated. Although different strains of B. amyloliquefaciens have a similar genome sequence, they have marked differences in the variable part of the genome (7); therefore, various strains of B. amyloliquefaciens may possess different abilities. Previous studies have demonstrated that numerous strains of B. amyloliquefaciens exert antifungal (8-10) and antibacterial activities (6,11-13). However, these studies have also indicated that various strains exert different antimicrobial activities. At present, several studies have suggested that $B$. amyloliquefaciens may be used as a potential therapeutic strategy that targets pathogens.

The major antimicrobial components of B. amyloliquefaciens are cyclic lipopeptides (CLPs) (14-16). At present, the three major CLPs that are known to possess antimicrobial activities are surfactin, iturin and fengycin A. Previous studies have demonstrated that surfactin, iturin and fengycin A possess antifungal and antibacterial 

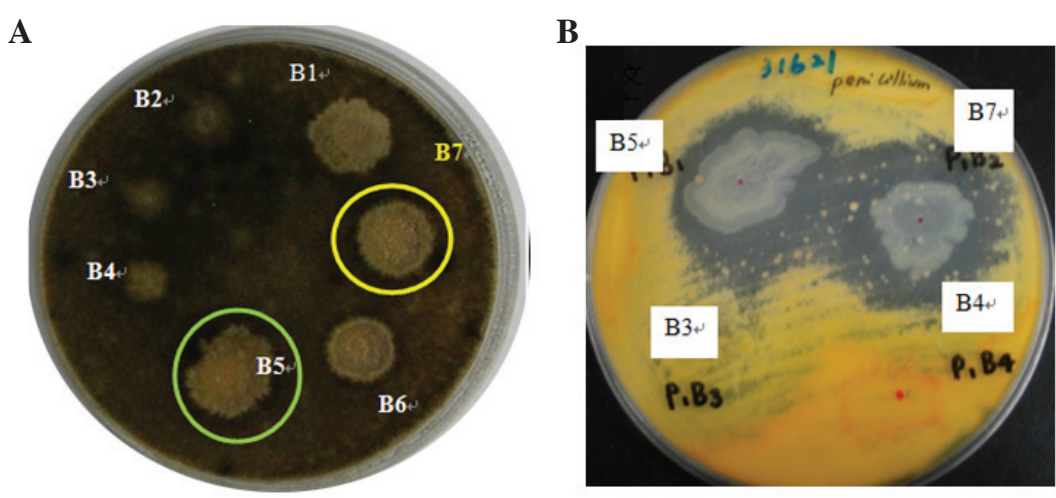

Figure 1. Screening of potential antimicrobial strains against (A) Aspergillus niger and (B) Penicillium pinophilum, as demonstrated from the zones of inhibition produced using the spot-on-the-lawn method.

activities (6,17-19). Notably, CLPs have several advantages, including low toxicity, high biodegradability and environmentally positive characteristics (20-23). These studies indicated that CLPs may be suitable for the treatment of pathogenic infections. In addition, other non-CLP antimicrobial peptides exist, such as amylolysin, which is a type of bacteriocin that is produced by $B$. amyloliquefacien $(24,25)$. The majority of bacteriocins only inhibit gram-positive bacteria, and certain non-CLPs are produced using the ribosomal synthesis method with gene modification, which is not the same as in the Bacillus genus (25). Therefore, CLPs isolated from B. amyloliquefaciens may be considered a better choice for the treatment of pathogenic infections, compared with non-CLPs.

Listeria monocytogenes is a foodborne pathogen, which induces listeriosis $(26,27)$. L. monocytogenes is present in several ready-to-eat foods, including vegetables, poultry and beef, which have been found to cause human listeriosis $(28,29)$. Due to clinical severity and the high mortality rates of L. monocytogenes-induced listeriosis, the control of $L$. monocytogenes food contamination is an important issue $(30,31)$. Previous studies have reported that bacteriocins produced by $B$. amyloliquefaciens can inhibit the growth of L. monocytogenes $(32,33)$. These studies indicated that products of $B$. amyloliquefaciens may be useful to control growth of L. monocytogenes; however, whether CLPs produced by $B$. amyloliquefaciens may target $L$. monocytogenes remains unclear.

Staphylococcus aureus can cause clinical acute hematogenous, osteomyelitis, endocarditis, pulmonary abscesses and spondylodiscitis (34-36). Methicillin-resistant $S$. aureus (MRSA) is not inhibited by treatment with several antibiotics $(37,38)$. MRSA is a major and serious pathogen, which is particularly associated with nosocomial infection $(39,40)$. Since numerous antibiotics can not effectively inhibit MRSA, the development of a novel anti-MRSA drug is important. A previous study indicated that $B$. amyloliquefaciens produced amylolysin, a ribosomally synthesized peptide, which can inhibit MRSA (25). However, to the best of our knowledge, whether CLPs produced by B. amyloliquefaciens can target MRSA has not yet been reported.

Based on the findings of previous studies, the present study aimed to determine whether CLPs isolated from
B. amyloliquefaciens purified from chicken intestines can exert antibacterial effects on MRSA and L. monocytogenes.

\section{Materials and methods}

Materials. Difco ${ }^{\mathrm{TM}}$ agar and Luria broth (LB) culture medium were obtained from BD Biosciences (Franklin Lakes, NJ, USA). The polymerase chain reaction (PCR) primers for $16 \mathrm{~S}$ ribosomal (r)RNA and DNA gyrase, subunit B (gyrB) genes were synthesized by MD Bio (Taipei, Taiwan). The API ${ }^{\circledR} 50$ CHB system assay kit was obtained from bioMerieux (Marcy-l'Étoile, France). Bond Elut ${ }^{\circledR} \mathrm{C} 18$ was purchased from Agilent Technologies (Santa Clara, CA, USA). The polytetrafluoroethylene membrane (JP020) was obtained from Advantec Co.,Ltd. (Tokyo, Japan). The indicator strains Staphylococcus epidermidis and Bacillus cereus were obtained from Bioresource Collection and Research Center (Hsinchu, Taiwan). The indicator strains Streptococcus pyogenes, Listeria monocytogenes, Clostridium tyrobutyricum, Escherichia coli, Helicobacter pylori, Salmonella typhimurium, Pseudomonas aeruginosa, Aspergillus flavus var. flavus, Aspergillus niger and Penicillium pinophilum were obtained from American Type Culture Collection (Manassas, VA, USA). MRSA HCT20 was kindly provided by the Department of Pathology and Laboratory Medicine, Taichung Veterans General Hospital (Taichung, Taiwan). The iturin A and surfactin standards were purchased from Sigma-Aldrich; Merck Millipore (Darmstadt, Germany).

Selection of potential bacterial strains from chicken intestines. Potential bacterial strains with antimicrobial activities isolated from chicken intestines (obtained from a local market, Taichung, Taiwan) using microscopic examination and streak plate methods were selected and determined using the modified spot-on-the-lawn method, as previously described $(41,42)$. Briefly, $0.7 \%$ top agar containing $10^{5}$ colony-forming units $(\mathrm{CFU}) / \mathrm{ml}$ indicator strains (A. niger or P. pinophilum) was overlaid onto a potato dextrose agar (PDA) plate, dried for $30 \mathrm{~min}$, and subsequently spotted with $2 \mu \mathrm{l}\left(10^{8} \mathrm{CFU} / \mathrm{ml}\right)$ of an overnight culture of the candidate strains. Subsequently, the PDA plates were incubated at $25^{\circ} \mathrm{C}$ for 5 days. Zones of inhibition are found in the sites containing potential strains with antimicrobial activities. 
Identification of potential bacterial strain. The B. amyloliquefaciens JN68 strain isolated from chicken intestines was identified by analyzing data from the API ${ }^{\circledR} 50 \mathrm{CHB}$ system $(43,44)$, as well as the results of a 16S rRNA and gyrB sequence detection (45-47).

The results were analyzed on the National Center for Biotechnology Information BLAST tool (blast.ncbi. nlm.nih.gov/Blast.cgi?PROGRAM=blastn\&PAGE_

TYPE=BlastSearch\&LINK_LOC=blasthome). According to morphological and physiological characteristics, biological testing and comparison of the $16 \mathrm{~S}$ rRNA sequence, the strain was identified as B. amyloliquefaciens. Briefly, phenotypic characterization and sugar fermentation pattern of the candidate strain was analyzed according to standard methods, using the $\mathrm{API}^{\circledR} 50 \mathrm{CHB}$ system assay kit. The obtained sugar fermentation pattern was analyzed using APILAB Plus software version 3.2.2 (bioMerieux). Further strain identification was confirmed by PCR detection of 16S rRNA and gyrB. Briefly, the candidate strain was cultured in LB culture medium $\left(30^{\circ} \mathrm{C}, 200 \times \mathrm{g}, 8-12 \mathrm{~h}\right)$ and the genomic DNA was extracted using the Blood and Tissue Genomic DNA Extraction Miniprep (Viogene BioTek Corporation, New Taipei City). The amplification of the 16S rRNA and gyrB gene region of candidate strain was conducted in a $50 \mu 1$ reaction mixture containing $1 \mu \mathrm{l}$ genomic DNA template, $5 \mu \mathrm{l} \mathrm{PCR}$ reaction buffer [10 mM Tris- $\mathrm{HCl}(\mathrm{pH} 8.8), 1.5 \mathrm{mM} \mathrm{MgCl}$, $50 \mathrm{mM} \mathrm{KCl}$ and $0.1 \%$ Triton $\mathrm{X}-100$ ] containing $200 \mu \mathrm{M}$ of each deoxynucleoside triphosphate (GE Healthcare Life Sciences, Chalfont, UK), $4 \mu 1 \mathrm{MgCl}_{2}$ (52 mM), $1 \mu \mathrm{M}$ each primer (Table I), 1 unit of Taq polymerase (GE Healthcare Life Sciences) and $39 \mu \mathrm{l}$ autoclaved Milli-Q water (Merck Millipore). The amplification was performed with thermocycling conditions as follows: Denaturation for $10 \mathrm{~min}$ at $95^{\circ} \mathrm{C}$; 35 cycles (as presented in Table I); termination at $72^{\circ} \mathrm{C}$ for 7 min. The PCR was conducted in a Robocycler ${ }^{\circledR}$ temperature thermal cycler (Agilent Technologies, Inc., Santa Clara, CA, USA). The PCR products were sequenced using an automatic sequencer (ABI PRISM ${ }^{\circledR}$ 3730; Applied Biosystems; Thermo Fisher Scientific, Inc., Waltham, MA, USA). Databases were screened for similarities using Basic Local Alignment Search Tool (48) and the alignment of overlapping fragments was performed using Vector NTI Advance 10 Contig Express software (Thermo Fisher Scientific, Inc.).

Culture conditions and isolation of the lipopeptide fraction. The B. amyloliquefaciens JN68 strain was cultured and activated with $5 \mathrm{ml} \mathrm{LB}$ culture medium at $30^{\circ} \mathrm{C}$. Subsequently, $5 \%$ B. amyloliquefaciens JN68 was seeded into $200 \mathrm{ml}$ number 3 medium (49). After incubation under an agitation rate of $\sim 200 \mathrm{rpm}$ at $30^{\circ} \mathrm{C}$ for 4 days, the fermentation medium was centrifuged at $11,000 \times \mathrm{g}$ for $20 \mathrm{~min}$ at $4^{\circ} \mathrm{C}$. The supernatant was collected and filtered through a polytetrafluoroethylene membrane $(0.22 \mu \mathrm{M}$ pore size). The filtered supernatant was then treated with $6 \mathrm{~N}$ $\mathrm{HCl}(\mathrm{pH} 2.0)$ at $4^{\circ} \mathrm{C}$ overnight, and was further centrifuged at $11,000 \times \mathrm{g}$ for $20 \mathrm{~min}$ at $4^{\circ} \mathrm{C}$. The sediment was washed with PBS and extracted three times with methanol. The methanolic extracts were concentrated and dissolved in methanol. Finally, the purified CLPs of the B. amyloliquefaciens JN68 strain were obtained from the methanolic extracts using the Bond Elut ${ }^{\circledR} \mathrm{C} 18$ (5 g) mini column as previously described (50).

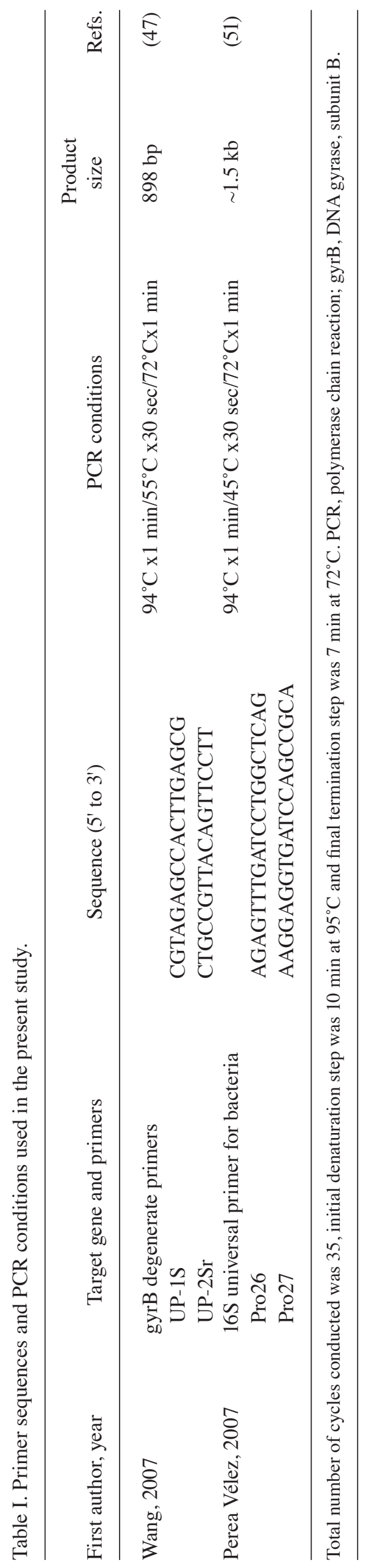


Identification of CLP structure by liquid chromatography-electrospray ionization-mass spectrometry (LC-ESI-MS)/MS. Purified CLPs produced by B. amyloliquefaciens JN68 were obtained from methanolic extracts as aforementioned. Subsequently, the structure of the CLPs was detected using LC and MS. For LC, an Agilent 1200 series system (Agilent Technologies), including a binary pump and an autosampler, was used for the chromatographic separation. The separation was conducted using an Atlantis C18 column $(150 \times 2.1 \mathrm{~mm}, 3.5 \mu \mathrm{m}$; Waters UK, Elstree, UK) at $30^{\circ} \mathrm{C}$ with a flow rate of $200 \mu \mathrm{l} / \mathrm{min}$ using the following gradient: i) $50 \% \mathrm{~B}$ for $2 \mathrm{~min}$; ii) a gradient of $50-100 \% \mathrm{~B}$ for $10 \mathrm{~min}$; iii) $100 \%$ B for $10 \mathrm{~min}$; iv) re-equilibration with $50 \%$ B for $10 \mathrm{~min}$. Mobile phase A consisted of $10 \%$ methanol and mobile phase B consisted of $100 \%$ methanol. The injection volume was set to $10 \mu 1$. For MS analysis an LTQ XL ${ }^{\mathrm{TM}}$ Linear Ion Trap MS was used (Thermo Fisher Scientific, Inc.), which was equipped with an ESI source. MS was operated in positive-ion mode with a spray voltage of $5.0 \mathrm{KV}$, and a capillary voltage of $40 \mathrm{~V}$. Capillary temperature and sheath gas $\left(\mathrm{N}_{2}\right)$ were set at $275^{\circ} \mathrm{C}$ and 20 arbitrary units, respectively. The precursor ion width was set at $3 \mathrm{Da}$ and the normalized collision energy was set at $35 \%$. For LC-ESI-MS/MS, the MS was operated in the selected target precursor ions as follows: $m / z$ 1,036, surfactin; 1,043, iturin A; 1464, fengycin A, using the Linear Ion Trap MS. The three major CLP components containing surfactin, iturin A and fengycin A were analyzed in the present study as previously described (52-54).

Antimicrobial profile assay. The antimicrobial activities of the purified CLPs produced by B. amyloliquefaciens JN68 were determined using an agar well diffusion assay, as previously described $(41,55)$. Briefly, small holes were dug into agar plates using a $200 \mu 1$ pipette tip. Each indicator microorganism $\left(\sim 10^{6} \mathrm{CFU} / \mathrm{ml}\right.$ bacterium or $10^{5} \mathrm{CFU} / \mathrm{ml}$ fungus) was spread onto an agar plate. Subsequently, $50 \mu 1$ B. amyloliquefaciens JN68 extracts were seeded into the holes on the agar plates for $2 \mathrm{~h}$ at $4^{\circ} \mathrm{C}$. The agar plates were then incubated for $16-18 \mathrm{~h}$ at $37^{\circ} \mathrm{C}$ (indicator bacteria) or at $30^{\circ} \mathrm{C}$ (indicator fungi). After a $16-18 \mathrm{~h}$ incubation, the appearance of the zones of inhibition was determined.

\section{Results}

Screening of potential antimicrobial strains. Several bacterial strains were obtained from the chicken intestines. In order to identify potential strains with antimicrobial activities, the spot-on-the-lawn method was used (41). In the present study, bacterial strains isolated from chicken intestines were spotted onto top agar plates containing indicator strains (A. niger or P. pinophilum). Zones of inhibition are found in the sites containing potential strains with antimicrobial activities. The results indicated that marked zones of inhibition appeared at the B5 and B7 sites; these sites therefore contained potential strains that can effectively inhibit the growth of A. niger (Fig. 1A) and P. pinophilum (Fig. 1B). Therefore, the strains at the B5 and B7 sites were considered to possess antimicrobial activities. In the present study, the potential bacterium at the B7 site was selected and analyzed (identical to B5).
Identification of the bacterial strain. The potential bacteria at the B7 site was primarily identified using the API ${ }^{\circledR} 50$ CHB system (Table II). Subsequently, the potential bacteria at the B7 site was further confirmed by $16 \mathrm{~S}$ rRNA and gyrB sequence detection. The PCR primer sequences of $16 \mathrm{~S}$ rRNA and gyrB used in the present study are presented in Table I. Analysis of the API ${ }^{\circledR} 50 \mathrm{CHB}$ system and gene sequence detection indicated that the potential bacteria at the B7 site belonged to the Bacillus amyloliquefaciens family. In the present study, this potential bacterial strain was named Bacillus amyloliquefaciens JN68.

Determination of CLP extract components. LC-ESI-MS/MS analysis of the CLP extracts is presented in Figs. 2-4. As shown in Fig. 2C, the main peak of surfactin lipopeptide was revealed at retention time $\left(R_{t}\right) 17.93$ min corresponding to protonated molecules $[\mathrm{M}+\mathrm{H}]^{+}$at $m / z, 1,036$ in positive modality. The representative MS/MS spectrum is shown in Fig. 2D. These results were similar to those observed during surfactin standard LC-ESI-MS/MS analysis (Fig. 2A and B). This analysis confirmed the presence of one main surfactin in the extracted CLPs. As shown in Fig. 3C, the one main peak of iturin A lipopeptide was revealed at $R_{t} 13.16$ min corresponding to the protonated molecules $[\mathrm{M}+\mathrm{H}]^{+}$at $\mathrm{m} / \mathrm{z}, 1,043$ in positive modality. The representative MS/MS spectrum is shown in Fig. 3D. The $R_{t}$ and product ions were similar to those obtained during iturin A standard LC-ESI-MS/MS analysis (Fig. 3A and B). The results of the LC-ESI-MS/MS analysis of fengycin A lipopeptide are presented in Fig. 4. The one main peak of fengycin A was observed at $R_{t} 13.90$ min corresponding to the protonated molecules $[\mathrm{M}+\mathrm{H}]^{+}$at $m / z, 1,464$ in positive modality (Fig. 4A). The representative MS/MS spectrum is shown in Fig. 4B. Although no fengycin A standard was used in the present study, the product ions were consistent with those from previous studies, which reported that product ions at $m / z, 1,080$ and 966 were representative ions present in the fengycin A MS/MS spectrum at m/z 1,464 (56,57). Taking into account the results obtained by LC-MS/MS analysis of CLPs, there are at least three lipopeptide groups within the whole B. amyloliquefaciens extract, which included surfactant, iturin A and fengycin A.

Antimicrobial spectrum of the potential strain. Previous studies have demonstrated that CLPs isolated from various B. amyloliquefaciens strains possess distinct antifungal $(9,17,58,59)$ and antibacterial activities (60-63). However, to the best of our knowledge, no previous experiment has demonstrated that CLPs isolated from B. amyloliquefaciens can inhibit MRSA and L. monocytogenes. The present study indicated that CLPs containing surfactin, iturin and fengycin A, isolated from B. amyloliquefaciens JN68, can inhibit several pathogenic fungal and bacterial strains (Table III). Notably, the present study is the first, to the best of our knowledge, to indicate that CLPs isolated from B. amyloliquefaciens JN68 can inhibit the growth of MRSA and L. monocytogenes (Table III).

\section{Discussion}

At present, various $B$. amyloliquefaciens strains have been isolated from plants and soil; in addition, some 
Table II. Sugar fermentation pattern of the candidate strain was determined using the API 50 CHB system.

Carbohydrate fermentation

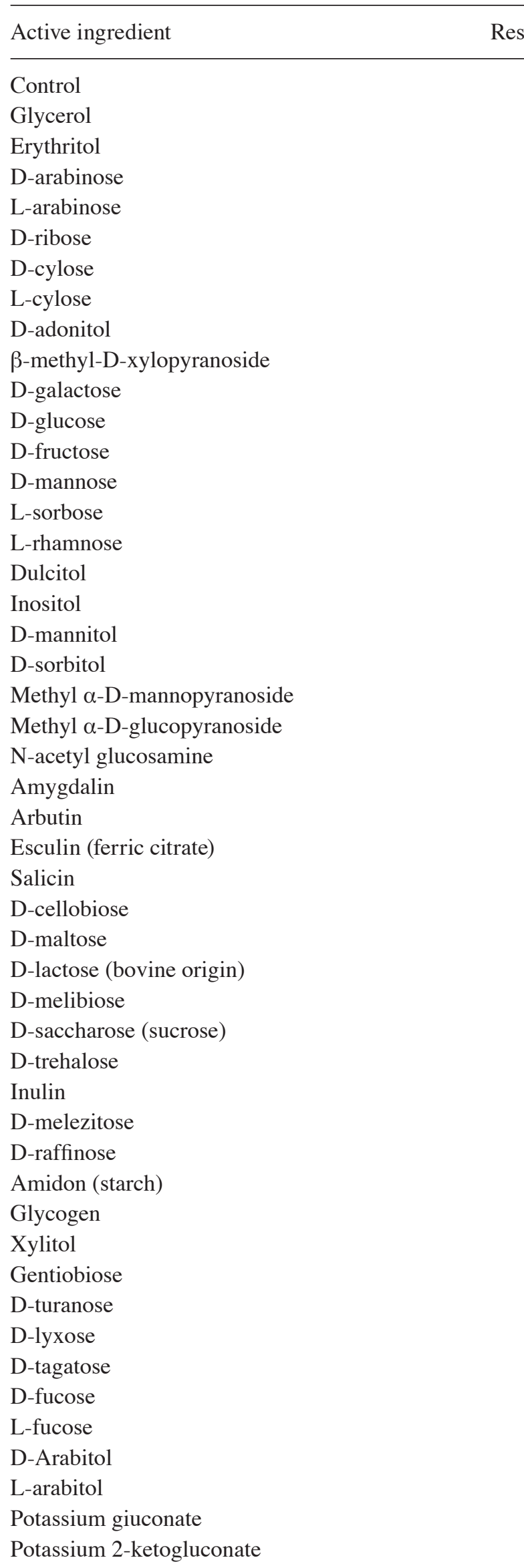

Table II. Continued.

Carbohydrate fermentation

Active ingredient

Response

Potassium 5-ketogluconate

Control is the culture medium alone. + , positive reaction; -, negative reaction; w, weak reaction.

B. amyloliquefaciens strains have been found to be present in animals (1-6). Several studies have reported that extracts of various $B$. amyloliquefaciens strains exert antimicrobial activities $(12,64,65)$. However, to the best of our knowledge, no previous studies have reported that $B$. amyloliquefaciens strains isolated from chickens possess antimicrobial activities. The present study isolated the B. amyloliquefaciens JN68 strain from chicken intestines, and confirmed it possessed antimicrobial activities. At present, several components have been identified that exert antimicrobial activities, including CLPs $(15,66,67)$, antifungal enzymes $(5,68)$ and non-CLPs $(24,25)$. Among these components, CLPs exert more broad-spectrum antifungal and antibacterial activities, compared with antifungal enzymes and non-CLPs $(17,64)$. In general, CLPs can exert antifungal and antibacterial activities (against both gram-positive and gram-negative bacteria) $(64,67)$, whereas antifungal enzymes only exert antifungal activities $(5,68)$ and non-CLPs exert only antibacterial activities against gram-positive bacteria $(24,25)$. In the present study, the CLP extracts purified from the B. amyloliquefaciens JN68 strain exerted antifungal and antibacterial activities on gram-positive and gram-negative bacteria.

Since CLPs produced by B. amyloliquefaciens possess several advantages, including broad-spectrum antimicrobial activities, low toxicity, high biodegradability and environmentally positive characteristics (20-23,64), CLPs may therefore be considered potential antimicrobials for food and clinical application. Previous studies have reported that surfactin, iturin and fengycin A are major CLP components that exert antimicrobial activities $(6,17-19)$. In the present study, the extracts purified from the B. amyloliquefaciens JN68 strain were confirmed to be surfactin, iturin and fengycin A. Since various $B$. amyloliquefaciens strains have similar genome sequences, with the exception of differences in the variable region of the genome (7), there are several types and expression levels of surfactin, iturin and fengycin A in the different strains (69). This may be why CLPs produced by different strains possess distinct antimicrobial activities.

Previous studies have reported that CLPs produced by some $B$. amyloliquefaciens strains possess antifungal activities, including the CGMCC5569, NJN-6, Q-426 and PPCB004 strains $(9,17,58,59)$. Furthermore, CLPs produced by some $B$. amyloliquefaciens strains possess antibacterial activities, including FZB42, HR62, NJN-6 and B9601-Y2 strains (60-63). In addition, CLPs produced by some B. amyloliquefaciens strains possess antifungal and antibacterial activities, such as the NJN-6 and B9601-Y2 strains $(9,62,63)$. 


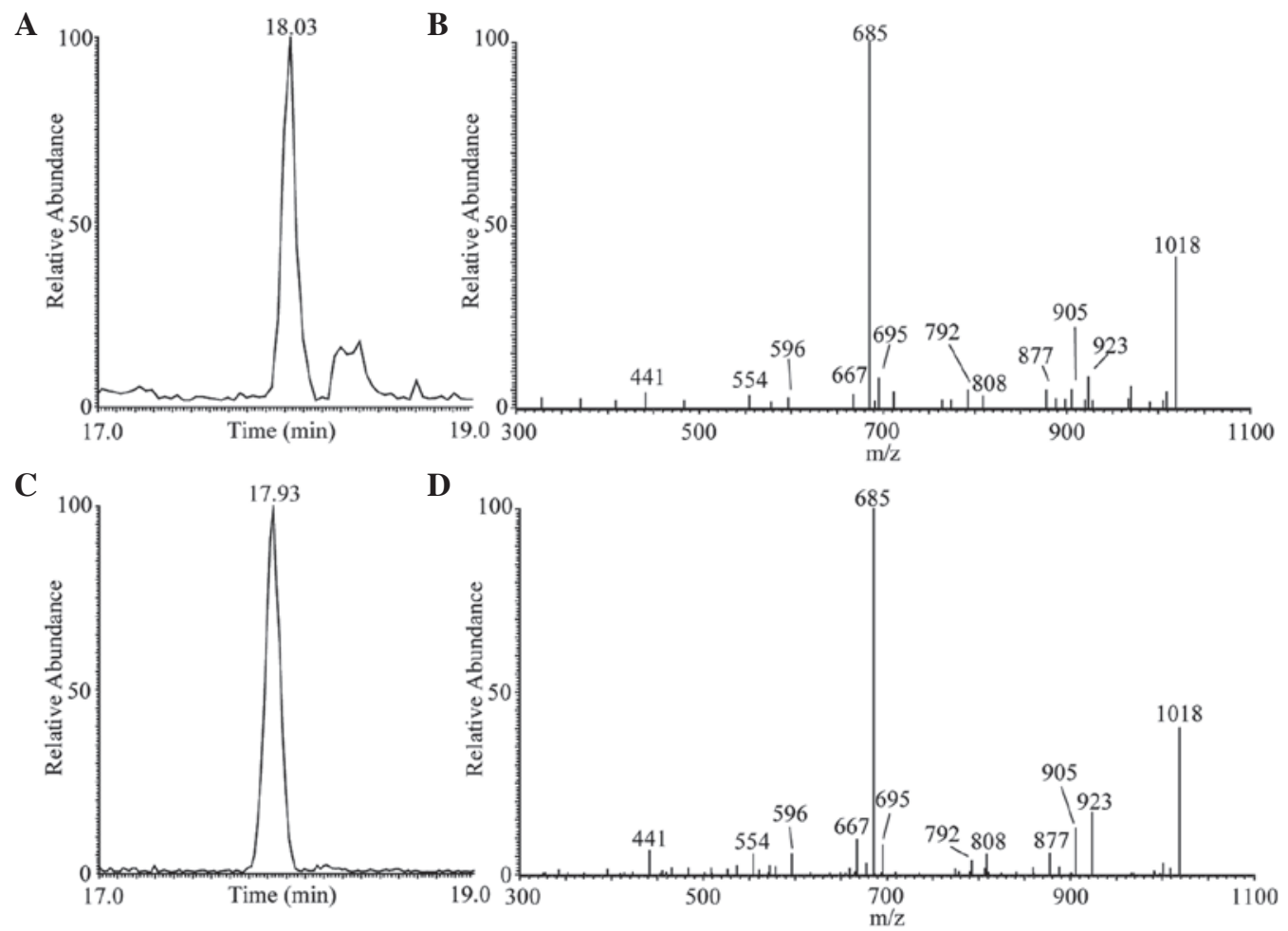

Figure 2. LC-ESI-MS/MS analysis of (A and B) surfactin standard and (C and D) biosurfactant lipopeptide extract. (A and C) Exact ion current chromatogram of surfactin $(\mathrm{m} / \mathrm{z}, 1,036)$. (B and D) Product ion spectra of the protonated molecules $[\mathrm{M}+\mathrm{H}]^{+}$of surfactin at $\mathrm{m} / \mathrm{z}, 1,036$. LC-ESI-MS/MS, liquid chromatography-electrospray ionization-mass spectrometry/mass spectrometry.

A

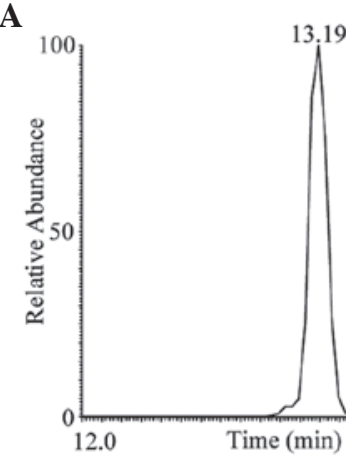

C

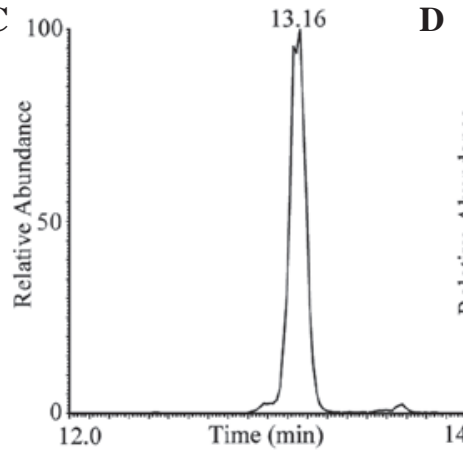

B
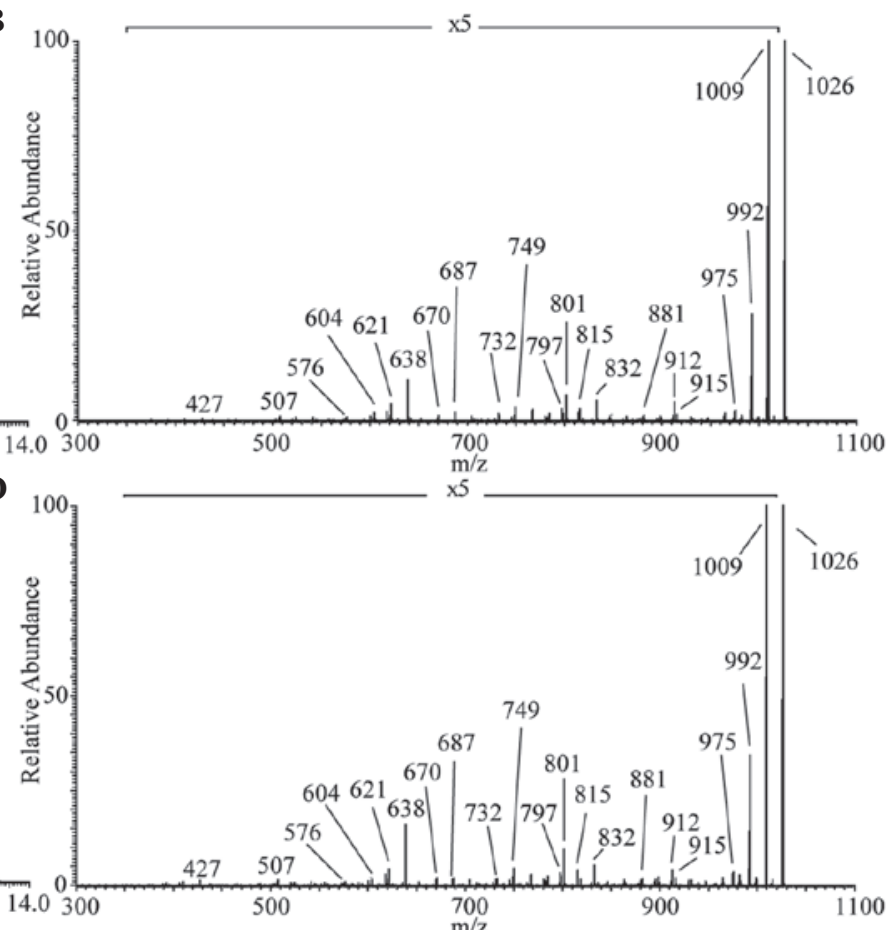

Figure 3. LC-ESI-MS/MS analysis of (A and B) iturin A standard and (C and D) biosurfactant lipopeptide extract. (A and C) Exact ion current chromatogram of iturin A $(\mathrm{m} / \mathrm{z}, 1,043)$. (B and D) Product ion spectra of the protonated molecules $[\mathrm{M}+\mathrm{H}]^{+}$of iturin A at $m / z$,, 043 . LC-ESI-MS/MS, liquid chromatography-electrospray ionization-mass spectrometry/mass spectrometry.

Compared with previous studies, antifungal and antibacterial activities were detected in the B. amyloliquefaciens JN68 strain isolated from chicken intestines in the present study. It is well known that MRSA is an important bacterial species that causes nosocomial infections $(39,40)$, whereas $L$. monocytogenes induces listeriosis, which has a high mortality rate $(30,31)$. No 
Table III. Antimicrobial spectrum of CLPs produced by Bacillus amyloliquefacients JN68 using the agar well diffusion method.

Antimicrobial activity ${ }^{\mathrm{a}}$

Indicator strain

Growth medium

Unpurified

Gram-positive

HCT20 MRSA

Staphylococcus epidermidis BCRC15245

Streptococcus pyogenes ATCC12344

Listeria monocytogenes ATCC15313

Clostridium tyrobutyricum ATCC25755

Bacillus cereus BCRC10250

Gram-negative

Escherichia coli ATCC11775 (10675)

Helicobacter pylori ATCC43526

Salmonella typhimurium ATCC13311

Pseudomonas aeruginosa ATCC9027 (11633)

Mold

Aspergillus flavus var. flavus $^{\mathrm{b}}$ ATCC 26770

Aspergillus niger ATCC16404

Penicillium pinophilum ATCC9644

$\begin{array}{cc}\text { TSA } & +++ \\ \text { NA } & ++ \\ \text { TSA with 5\% blood } & +++ \\ \text { BHI } & +++ \\ \text { NA } & + \\ \text { NA } & + \\ & \\ \text { NA } & ++ \\ \text { Brucella with 3\% blood } & + \\ \text { TSA } & +++ \\ \text { NA } & ++ \\ \text { PDA } & \\ \text { PDA } & +++ \\ \text { PDA } & + \\ & +++\end{array}$

anterpretation of zone of inhibition diameter:,$+ 5-10 \mathrm{~mm}$ (weak inhibition); ++, 10-15 mm (moderate inhibition); +++, >15 mm (strong

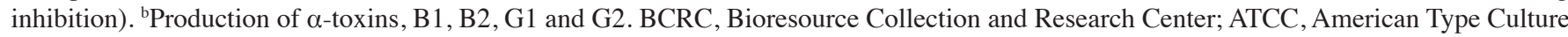
Collection; MRSA, methicillin-resistant Staphylococcus aureus; TSA, tryptone soya agar; NA, nutrient agar; BHI, brain-heart infusion medium; PDA, potato dextrose agar.

A

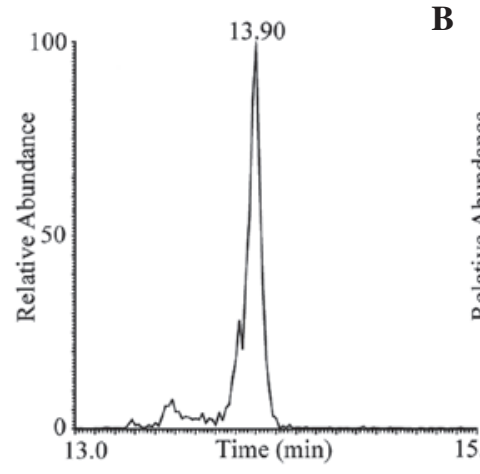

B

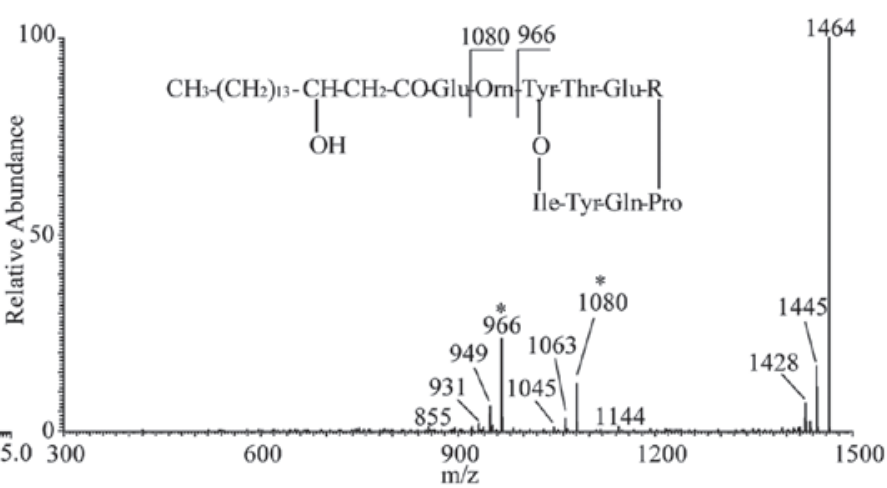

Figure 4. LC-ESI-MS/MS analysis of biosurfactant lipopeptide extract. (A) Exact ion current chromatogram of fengycin A ( $m / z$, 1,464). (B) Product ion spectra of the protonated molecules $[\mathrm{M}+\mathrm{H}]^{+}$of fengycin A at $\mathrm{m} / \mathrm{z}, 1,464$. Significant fragment ions of fengycin A were marked with an asterisk. LC-ESI-MS/MS, liquid chromatography-electrospray ionization-mass spectrometry/mass spectrometry.

previous studies, to the best of our knowledge, have demonstrated that CLPs produced by B. amyloliquefaciens can inhibit the growth of MRSA and L. monocytogenes. Therefore, the present study is the first to indicate that the B. amyloliquefaciens JN68 strain can inhibit MRSA and L. monocytogenes.

In conclusion, the present study isolated B. amyloliquefaciens JN68 from chicken intestines, and confirmed it possessed antifungal and antibacterial activities. In particular, B. amyloliquefaciens JN68 was able to inhibit the growth of MRSA and L. monocytogenes. These results suggested that the B. amyloliquefaciens JN68 strain may be applied to prevent the occurrence of foodborne diseases and nosocomial infections.

\section{Acknowledgements}

The present study was supported by grants from the Taipei Tzu Chi Hospital (grant nos. TCRD-TPE-104-34 and TCRD-TPE-104-06).

\section{References}

1. Niazi A, Manzoor S, Bejai S, Meijer J and Bongcam-Rudloff E: Complete genome sequence of a plant associated bacterium Bacillus amyloliquefaciens subsp. plantarum UCMB5033. Stand Genomic Sci 9: 718-725, 2014. 
2. Cai J, Liu F, Liao X and Zhang R: Complete genome sequence of Bacillus amyloliquefaciens LFB112 isolated from Chinese herbs, a strain of a broad inhibitory spectrum against domestic animal pathogens. J Biotechnol 175: 63-64, 2014.

3. Ait Kaki A, Kacem Chaouche N, Dehimat L, Milet A, Youcef-Ali M, Ongena $\mathrm{M}$ and Thonart P: Biocontrol and plant growth promotion characterization of Bacillus species isolated from calendula officinalis rhizosphere. Indian J Microbiol 53: 447-452, 2013.

4. Geetha I, Aruna R and Manonmani AM: Mosquitocidal Bacillus amyloliquefaciens: Dynamics of growth \& production of novel pupicidal biosurfactant. Indian J Med Res 140: 427-434, 2014

5. Han JH, Shim H, Shin JH and Kim KS: Antagonistic activities of Bacillus spp. strains isolated from tidal flat sediment towards anthracnose pathogens Colletotrichum acutatum and C. gloeosporioides in South Korea. Plant Pathol J 31: 165-175, 2015.

6. Xu HM, Rong YJ, Zhao MX, Song B and Chi ZM: Antibacterial activity of the lipopetides produced by Bacillus amyloliquefaciens M1 against multidrug-resistant Vibrio spp. isolated from diseased marine animals. Appl Microbiol Biotechnol 98: 127-136, 2014.

7. Ruckert C, Blom J, Chen X, Reva O and Borriss R: Genome sequence of B. amyloliquefaciens type strain DSM7(T) reveals differences to plant-associated B. amyloliquefaciens FZB42. J Biotechnol 155: 78-85, 2011.

8. Xu Z, Shao J, Li B, Yan X, Shen Q and Zhang R: Contribution of bacillomycin D in Bacillus amyloliquefaciens SQR9 to antifunga activity and biofilm formation. Appl Environ Microbiol 79: 808-815, 2013.

9. Yuan J, Raza W, Huang Q and Shen Q: The ultrasound-assisted extraction and identification of antifungal substances from B.amylo liquefaciens strain NJN-6 suppressing Fusarium oxysporum.J Basic Microbiol 52: 721-730, 2012

10. Zhao P, Quan C, Jin L, Wang L, Wang J and Fan S: Effects of critical medium components on the production of antifungal lipopeptides from Bacillus amyloliquefaciens Q-426 exhibiting excellent biosurfactant properties. World J Microbiol Biotechnol 29: 401-409, 2013

11. Chi Z, Rong YJ, Li Y, Tang MJ and Chi ZM: Biosurfactins production by Bacillus amyloliquefaciens $\mathrm{R} 3$ and their antibacteria activity against multi-drug resistant pathogenic E. coli. Bioprocess Biosyst Eng 38: 853-861, 2015

12. Kadaikunnan S, Rejiniemon T, Khaled JM, Alharbi NS and Mothana R: In-vitro antibacterial, antifungal, antioxidant and functional properties of Bacillus amyloliquefaciens. Ann Clin Microbiol Antimicrob 14: 9, 2015

13. Cao H, He S, Wei R, Diong M and Lu L: Bacillus amyloliquefaciens G1: A potential antagonistic bacterium against eel-pathogenic aeromonas hydrophila. Evid Based Complement Alternat Med 2011: 824104, 2011.

14. Ma Z, Hu J, Wang X and Wang S: NMR spectroscopic and MS/MS spectrometric characterization of a new lipopeptide antibiotic bacillopeptin B1 produced by a marine sediment-derived Bacillus amyloliquefaciens SH-B74. J Antibiot (Tokyo) 67: 175-178, 2014.

15. Mora I, Cabrefiga J and Montesinos E: Cyclic lipopeptide biosynthetic genes and products, and inhibitory activity of plant-associated Bacillus against phytopathogenic bacteria. PLoS One 10: e0127738, 2015.

16. Nihorimbere V, Cawoy H, Seyer A, Brunelle A, Thonart P and Ongena M: Impact of rhizosphere factors on cyclic lipopeptide signature from the plant beneficial strain Bacillus amyloliquefaciens S499. FEMS Microbiol Ecol 79: 176-191, 2012.

17. Arrebola E, Jacobs R and Korsten L: Iturin A is the principal inhibitor in the biocontrol activity of Bacillus amyloliquefaciens PPCB004 against postharvest fungal pathogens. J Appl Microbiol 108: 386-395, 2010.

18. Arguelles-Arias A, Ongena M, Halimi B, Lara Y, Brans A, Joris B and Fickers P: Bacillus amyloliquefaciens GA1 as a source of potent antibiotics and other secondary metabolites for biocontrol of plant pathogens. Microb Cell Fact 8: 63. 2009.

19. Vágvölgyi C, Sajben-Nagy E, Bóka B, Vörös M, Berki A, Palágyi A, Krisch J, Skrbić B, Durišić-Mladenović N and Manczinger L: Isolation and characterization of antagonistic Bacillus strains capable to degrade ethylenethiourea. Curr Microbiol 66: 243-250, 2013.

20. Kim PI, Bai H, Bai D, Chae H, Chung S, Kim Y, Park R and Chi YT: Purification and characterization of a lipopeptide produced by Bacillus thuringiensis CMB26. J Appl Microbiol 97: 942-949, 2004

21. Maget-Dana R and Peypoux F: Iturins, a special class of pore-forming lipopeptides: Biological and physicochemical properties. Toxicology 87: 151-174, 1994.
22. Stein T: Bacillus subtilis antibiotics: Structures, syntheses and specific functions. Mol Microbiol 56: 845-857, 2005 .

23. Yoshida S, Hiradate S, Tsukamoto T, Hatakeda K and Shirata A Antimicrobial activity of culture filtrate of Bacillus amyloliquefaciens RC-2 isolated from mulberry leaves. Phytopathology 91 181-187, 2001.

24. Benitez L, Correa A, Daroit D and Brandelli A: Antimicrobial activity of Bacillus amyloliquefaciens LBM 5006 is enhanced in the presence of Escherichia coli. Curr Microbiol 62: 1017-1022, 2011.

25. Arguelles Arias A, Ongena M, Devreese B, Terrak M, Joris B and Fickers P: Characterization of amylolysin, a novel lantibiotic from Bacillus amyloliquefaciens GA1. PLoS One 8: e83037, 2013.

26. Salazar JK, Wu Z, McMullen PD, Luo Q, Freitag NE, Tortorello ML, Hu S and Zhang W: PrfA-like transcription factor gene lmo0753 contributes to L-rhamnose utilization in Listeriamonocytogenes strains associated with human food-borne infections. Appl Environ Microbiol 79: 5584-5592, 2013.

27. Shalaby MA, Mohamed MS, Mansour MA and Abd El-Haffiz AS: Comparison of polymerase chain reaction and conventional methods for diagnosis of Listeria monocytogenes isolated from different clinical specimens and food stuffs. Clin Lab 57: 919-924, 2011

28. Neetoo H, Ye M and Chen H: Potential antimicrobials to control Listeria monocytogenes in vacuum-packaged cold-smoked salmon pâté and fillets. Int J Food Microbiol 123: 220-227, 2008.

29. Pérez-Rodriguez F, van Asselt ED, Garcia-Gimeno RM, Zurera $\mathrm{G}$ and Zwietering MH: Extracting additional risk managers information from a risk assessment of Listeria monocytogenes in deli meats. J Food Prot 70: 1137-1152, 2007.

30. Althaus D, Lehner A, Brisse S, Maury M, Tasara T and Stephan R: Characterization of Listeria monocytogenes strains isolated during 2011-2013 from human infections in Switzerland. Foodborne Pathog Dis 11: 753-758, 2014.

31. Pontello M, Guaita A, Sala G, Cipolla M, Gattuso A, Sonnessa M and Gianfranceschi MV: Listeria monocytogenes serotypes in human infections (Italy, 2000-2010). Ann Ist Super Sanita 48: 146-150, 2012

32. Lisboa MP, Bonatto D, Bizani D, Henriques JA and Brandelli A: Characterization of a bacteriocin-like substance produced by Bacillus amyloliquefaciens isolated from the Brazilian Atlantic forest. Int Microbiol 9: 111-118, 2006

33. Sağdiç O, Ozkan G, Ozcan M and Ozçelik S: A study on inhibitory effects of Siğla tree (Liquidambar orientalis Mill. var. orientalis) storax against several bacteria. Phytother Res 19: 549-551, 2005.

34. Agarwal A and Aggarwal AN: Bone and joint infections in children: Acute hematogenous osteomyelitis. Indian J Pediatr, 2015.

35. Christiansen JG, Jensen HE, Johansen LK, Kochl J, Koch J, Aalbaek B, Nielsen OL and Leifsson PS: Porcine models of non-bacterial thrombotic endocarditis (NBTE) and infective endocarditis (IE) caused by Staphylococcus aureus: A preliminary study. J Heart Valve Dis 22: 368-376, 2013.

36. Vos FJ, Kullberg BJ, Sturm PD, Krabbe PF, van Dijk AP, Wanten GJ, Oyen WJ and Bleeker-Rovers CP: Metastatic infectious disease and clinical outcome in Staphylococcus aureus and Streptococcus species bacteremia. Medicine (Baltimore) 91: 86-94, 2012.

37. Hasanvand A, Ghafourian S, Taherikalani M, Jalilian FA, Sadeghifard N and Pakzad I: Antiseptic resistance in methicillin sensitive and methicillin resistant Staphylococcus aureus isolates from some major hospitals, Iran. Recent Pat Antiinfect Drug Discov 10: 105-112, 2015.

38. Kaur DC and Chate SS: Study of antibiotic resistance pattern in methicillin resistant Staphylococcus aureus with special reference to newer antibiotic. J Glob Infect Dis 7: 78-84, 2015.

39. McMaster J, Booth MG, Smith A and Hamilton K: Meticillin-resistant Staphylococcus aureus in the intensive care unit: Its effect on outcome and risk factors for acquisition. J Hosp Infect 90: 327-332, 2015.

40. Dancer SJ: Controlling hospital-acquired infection: Focus on the role of the environment and new technologies for decontamination. Clin Microbiol Rev 27: 665-690, 2014.

41. Lima ET, Andreatti Filho RL, Okamoto AS, Noujaim JC, Barros MR and Crocci AJ: Evaluation in vitro of the antagonistic substances produced by Lactobacillus spp. isolated from chickens. Can J Vet Res 71: 103-107, 2007. 
42. Chen H, Wang L, Su CX, Gong GH, Wang P and Yu ZL: Isolation and characterization of lipopeptide antibiotics produced by Bacillus subtilis. Lett Appl Microbiol 47: 180-186, 2008.

43. Østensvik Ø, From C, Heidenreich B, O'Sullivan K and Granum PE: Cytotoxic Bacillus spp. belonging to the B. cereus and B. subtilis groups in Norwegian surface waters. J Appl Microbiol 96: 987-993, 2004.

44. Zaghloul TI, Al-Bahra M and Al-Azmeh $\mathrm{H}$ : Isolation, identification, and keratinolytic activity of several feather-degrading bacterial isolates. Appl Biochem Biotechnol 70-72: 207-213, 1998.

45. Marroki A, Zúñiga $M$, Kihal $M$ and Pérez-Martinez G: Characterization of lactobacillus from algerian goat's milk based on phenotypic, $16 \mathrm{~S}$ rDNA sequencing and their technological properties. Braz J Microbiol 42: 158-171, 2011.

46. Tajbakhsh M, Nayer BN, Motavaze K, Kharaziha P, Chiani M, Zali MR and Klena JD: Phylogenetic relationship of Salmonella enterica strains in Tehran, Iran, using 16S rRNA and gyrB gene sequences. J Infect Dev Ctries 5: 465-472, 2011.

47. Wang LT, Lee FL, Tai CJ and Kasai H: Comparison of gyrB gene sequences, 16S rRNA gene sequences and DNA-DNA hybridization in the Bacillus subtilis group. Int J Syst Evol Microbiol 57: 1846-1850, 2007.

48. Altschul SF, Madden TL, Schäffer AA, Zhang J, Zhang Z, Miller W and Lipman DJ: Gapped BLAST and PSI-BLAST: A new generation of protein database search programs. Nucleic Acids Res 25: 3389-3402, 1997.

49. Tsuge K, Akiyama T and Shoda M: Cloning, sequencing, and characterization of the iturin A operon. J Bacteriol 183: 6265-6273, 2001

50. Razafindralambo H, Paquot M, Hbid C, Jacques P, Destain J and Thonart P: Purification of antifungal lipopeptides by reversed-phase high-performance liquid chromatography. J Chromatogr 639: 81-85, 1993.

51. Perea Vélez M,Hermans K, Verhoeven TL,Lebeer SE, Vanderleyden $\mathrm{J}$ and De Keersmaecker SC: Identification and characterization of starter lactic acid bacteria and probiotics from Columbian dairyproducts. J Appl Microbiol 103: 666-674, 2007.

52. Zeriouh H, de Vicente A, Pérez-Garcia A and Romero D: Surfactin triggers biofilm formation of Bacillus subtilis in melon phylloplane and contributes to the biocontrol activity. Environ Microbiol 16 2196-2211, 2014.

53. Zhao X, Han Y, Tan XQ, Wang J and Zhou ZJ: Optimization of antifungal lipopeptide production from Bacillus sp. BH072 by response surface methodology. J Microbiol 52: 324-332, 2014.

54. Wang J, Liu J, Wang X, Yao J and Yu Z: Application of electrospray ionization mass spectrometry in rapid typing of fengycin homologues produced by Bacillus subtilis. Lett Appl Microbiol 39: 98-102, 2004

55. Tagg JR and McGiven AR: Assay system for bacteriocins. Appl Microbiol 21: 943, 1971

56. Pecci Y, Rivardo F, Martinotti MG and Allegrone G: LC/ESI-MS/MS characterisation of lipopeptide biosurfactants produced by the Bacillus licheniformis V9T14 strain. J Mass Spectrom 45: 772-778, 2010.

57. Vanittanakom N, Loeffler W, Koch U and Jung G: Fengycin-a novel antifungal lipopeptide antibiotic produced by Bacillus subtilis F-29-3. J Antibiot (Tokyo) 39: 888-901, 1986.
58. Zhao P, Quan C, Wang Y, Wang J and Fan S: Bacillus amyloliquefaciens Q-426 as a potential biocontrol agent against Fusarium oxysporum f. sp. spinaciae. J Basic Microbiol 54: 448-456, 2014

59. Yuan B, Wang Z, Qin S, Zhao GH, Feng YJ, Wei LH and Jiang JH Study of the anti-sapstain fungus activity of Bacillus amyloliquefaciens CGMCC 5569 associated with Ginkgo biloba and identification of its active components. Bioresour Technol 114: 536-541, 2012.

60. Kröber M, Wibberg D, Grosch R, Eikmeyer F, Verwaaijen B, Chowdhury SP, Hartmann A, Pühler A and Schlüter A: Effect of the strain Bacillus amyloliquefaciens FZB42 on the microbial community in the rhizosphere of lettuce under field conditions analyzed by whole metagenome sequencing. Front Microbiol 5: 252,2014

61. Huang J, Wei Z, Tan S, Mei X, Shen Q and Xu Y: Suppression of bacterial wilt of tomato by bioorganic fertilizer made from the antibacterial compound producing strain Bacillus amyloliquefaciens HR62. J Agric Food Chem 62: 10708-10716, 2014.

62. Yuan J, Zhang F, Wu Y, Zhang J, Raza W, Shen Q and Huang Q: Recovery of several cell pellet-associated antibiotics produced by Bacillus amyloliquefaciens NJN-6. Lett Appl Microbiol 59: 169-176, 2014.

63. He P, Hao K, Blom J, Rückert C, Vater J, Mao Z, Wu Y, Hou M, He P, He Y and Borriss R: Genome sequence of the plant growth promoting strain Bacillus amyloliquefaciens subsp. plantarum B9601-Y2 and expression of mersacidin and other secondary metabolites. J Biotechnol 164: 281-291, 2012.

64. Compaoré CS, Nielsen DS, Sawadogo-Lingani H, Berner TS, Nielsen KF, Adimpong DB, Diawara B, Ouédraogo GA, Jakobsen M and Thorsen L: Bacillus amyloliquefaciens ssp. plantarum strains as potential protective starter cultures for the production of Bikalga, an alkaline fermented food. J Appl Microbiol 115: 133-146, 2013

65. Hajji S, Ghorbel-Bellaaj O, Younes I, Jellouli K and Nasri M: Chitin extraction from crab shells by Bacillus bacteria. Biological activities of fermented crab supernatants. Int J Biol Macromol 79: 167-173, 2015.

66. Alvarez F, Castro M, Principe A, Borioli G, Fischer S, Mori G and Jofré E: The plant-associated Bacillus amyloliquefaciens strains MEP2 18 and ARP2 3 capable of producing the cyclic lipopeptides iturin or surfactin and fengycin are effective in biocontrol of sclerotinia stem rot disease. J Appl Microbiol 112: 159-174, 2012.

67. Hsieh FC, Lin TC, Meng M and Kao SS: Comparing methods for identifying Bacillus strains capable of producing the antifungal lipopeptide iturin A. Curr Microbiol 56: 1-5, 2008.

68. Wang SL, Shih IL, Liang TW and Wang CH: Purification and characterization of two antifungal chitinases extracellularly produced by Bacillus amyloliquefaciens V656 in a shrimp and crab shell powder medium. J Agric Food Chem 50: 2241-2248, 2002.

69. Ongena M and Jacques P: Bacillus lipopeptides: Versatile weapons for plant disease biocontrol. Trends Microbiol 16 115-125, 2008. 